\title{
Complex nature of serum lysozyme activity: evidence of thermolability in inflammatory bowel disease
}

\author{
M. WARD, W. D. MITCHELL, AND M. EASTWOOD \\ From the Gastro-intestinal Unit and Department of Biochemistry, Western General Hospital and \\ University of Edinburgh, Edinburgh, UK
}

SUMMARY In patients with Crohn's disease and ulcerative colitis, alterations in serum storage temperature produced significant changes in serum lysozyme activity (SLA) as measured by the lysoplate method. This was not the case in healthy controls or in a group with other gastrointestinal disorders. Electrophoretic separation of serum revealed two components of lysozyme-type lytic activity but only one in extracts of gut mucosa, leucocytes, and egg white. The major lytic component of serum migrated towards the cathode and reacted with specific antilysozyme serum, but the minor component which migrated towards the anode did not. Although the cause of this anionic lytic activity is uncertain, it contributes to total serum activity as estimated by any method utilising the lysis of Micrococcus lysodeikticus, and may possibly be related to the observed thermolability.

Measurements of serum lysozyme activity (SLA) are of relevance to several specialties including haematology, oncology, and nephrology (Osserman et al., 1974). More recently, gastroenterology has been added to this list with a report in which increased SLA was described in Crohn's disease and contrasted with normal activity in patients with ulcerative colitis (Falchuk et al., 1975). In subsequent studies, however, the absolute distinction of Crohn's disease patients from normal subjects and from those with ulcerative colitis has not been confirmed (Dronfield and Langman, 1975; Peeters et al., 1976; Nugent et al., 1976; Mallas et al., 1976).

In the course of an evaluation of the value of SLA in gastrointestinal disorders, it became apparent that human serum lysozyme did not possess the characteristic thermostability of purified animal lysozyme (Jolles, 1962). The thermostability of serum lysozyme has, therefore, been studied in various gastrointestinal disorders, and the electrophoretic mobility of lysozyme activity has been investigated.

\section{Methods}

PATIENTS AND CONTROLS

Eighteen patients with Crohn's disease (11 female, 7 male, mean age 38.6 years) and 12 patients with ulcerative colitis ( 6 female, 6 male, mean age 40.4 years) were studied. The diagnosis and distribution

Received for publication 10 August 1977 and activity of the disease were determined by the usually accepted clinical, radiological, and histological criteria (Lennard-Jones et al., 1968; Schachter and Kirsner, 1975). Thirteen healthy laboratory and clinical staff were used as controls ( 7 male, 6 female, mean age 32.0 years). A further 'miscellaneous GI disease' group was investigated, comprising five patients with active duodenal ulceration, confirmed by endoscopy, and four patients with functional gastrointestinal disorders (4 female, 5 male, mean age $36 \cdot 0$ years).

SERUM COLLECTION AND STORAGE

The venous blood was allowed to coagulate at room temperature (approximately $20^{\circ} \mathrm{C}$ ) for two to three hours and separated, and SLA was measured on aliquots immediately after separation (fresh) and then after four days' storage at room temperature, $+6^{\circ} \mathrm{C}$, and $-20^{\circ} \mathrm{C}$.

TISSUE/LEUCOCYTE EXTRACTS

For comparison of the electrophoretic mobility of tissue and other sources of lysozyme with that of serum, mucosal samples obtained at endoscopy of gastric, small bowel, and rectal tissue were homogenised in maleate buffer, $\mathrm{pH} 6.8$, and filtered. Leucocytes obtained from a normal volunteer were separated by $5 \%$ Dextran sedimentation of heparinised whole blood and disrupted by six cycles of freezing and thawing. The resulting suspensions were filtered, and lysozyme activity was determined in the filtrate. 
ESTIMATION OF SLA

The lysoplate method of Osserman and Lawlor (1966) was used. Disease and control samples were included in each plate together with standards and readings made in duplicate. Values for test samples were calculated from a semilog arithmic standard curve. Standards used were either egg white lysozyme (EWL) or human lysozyme.

\section{ELECTROPHORETIC SEPARATION OF SLA}

\section{(i) Agarose}

Two per cent agarose (phosphate buffer pH 7.2) was poured onto glass slides, and $10 \mu \mathrm{l}$ wells were cut into the solidified agarose. Standards and test samples were applied and electrophoresed at $30 \mathrm{~mA}$ for two hours. Each plate was then overlaid with a preformed indicator layer of Micrococcus lysodeikticus (pH 6.3, $50 \mathrm{mg}$ organisms $/ 100 \mathrm{ml} 2 \%$ agarose). This combination was then incubated for 18 hours at $37^{\circ} \mathrm{C}$ to determine zones of lytic activity.

\section{(ii) Micrococcus}

In an attempt to improve the definition of the lytic zones formed by method (i) and to develop a semiquantitative method, electrophoresis was performed through a micrococcus-agarose layer (pH 6.3, $50 \mathrm{mg}$ organisms $/ 100 \mathrm{ml} 2 \%$ agarose) for two hours at 30 $\mathbf{m A}$. The agarose layers were subsequently air dried at $60^{\circ} \mathrm{C}$ for one hour to provide a permanent record of the resulting lytic zones.

\section{(iii) Immunoelectrophoresis}

Electrophoresis of serum was performed in $2 \%$ agarose in $0.05 \mathrm{M}$ barbital buffer $\mathrm{pH} 8.2$ for two hours at $12 \mathrm{~mA}$. Anti-human lysozyme serum (Hoechst-Behring) was then poured into a trough cut parallel to the axis of electrophoresis, and the plate was allowed to develop for $\mathbf{4 8}$ hours in a moist chamber.

\section{(iv) Radioimmunoelectrophoresis}

After a preliminary electrophoresis similar to that of (iii) the plate was overlaid with a solution of antihuman lysozyme serum previously labelled by the chloramine-T method with radioactive ${ }^{125} \mathrm{I}$. After a period of $\mathbf{4 8}$ hours allowed for interaction the plate was washed with five changes of buffer, and autoradiography was performed to determine the location of lysozyme activity.

\section{Results}

EFFECTS OF TEMPERATURE OF STORAGE ON LYSOPLATE VALUES

Figure 1 shows the results of repeat measurement of
SLA after four days' storage at one of three temperatures. Statistical comparison using paired $t$ tests was performed and the $P$ values in comparison with the value for the 'fresh' sample are shown in the Figure. The most striking finding was the significant drop in SLA with storage temperature for the ulcerative colitis patients. There was a significant drop in SLA of Crohn's patients at $-20^{\circ} \mathrm{C}$ storage and a significant rise in the room temperature specimens. No significant change occurred in the SLA of normal controls nor in the miscellaneous group. If all results are plotted together, however (Fig. 2), then the separation of control and disease populations apparent in the fresh and room temperature estimates is lost in those from the $-20^{\circ} \mathrm{C}$ samples. Neither disease activity nor distribution appeared to influence SLA stability in the patients with inflammatory bowel disease, and in the miscellaneous group subjects with active pepticulceration did not differ in this respect from those with functional disorders.

\section{ELECTROPHORETIC SEPARATION}

\section{(i) Agarose}

In all samples (serum standards, mucosal and leucocyte) possessing lysozyme activity, such activity was found to have migrated towards the cathode. Serum alone, however, showed additional anionic lytic activity. It was noted that this anionic lytic activity was more pronounced if the sample had been stored for more than 20 hours at room temperature.

\section{(ii) Micrococcus}

When electrophoresis was performed through agarose containing micrococcus, the anticipated cationic lytic zones developed in all lysozyme-containing extracts. Only serum showed definite anionic activity whereas purified human lysozyme showed very faint anionic lytic activity (Fig. 3). It was also found that the distance between the origin and the peak of the cationic lytic zone bore a semilogarithmic relationship to the concentration of EWL standards. When this value was calculated for serum it was found always to be less than that calculated for the same sample from the lysoplate, whereas values by both methods for other sources of lysozyme were similar. This suggests that the 'total' serum lysozyme activity as measured by the lysoplate represents the combined effects of anionic and cationic components. As the distance migrated by the anionic component was not related to concentration, its contribution could not be measured directly. It could be estimated, however, by subtracting the value calculated for the cationic component from that for the same sample by lysoplate, relating both values to EWL 


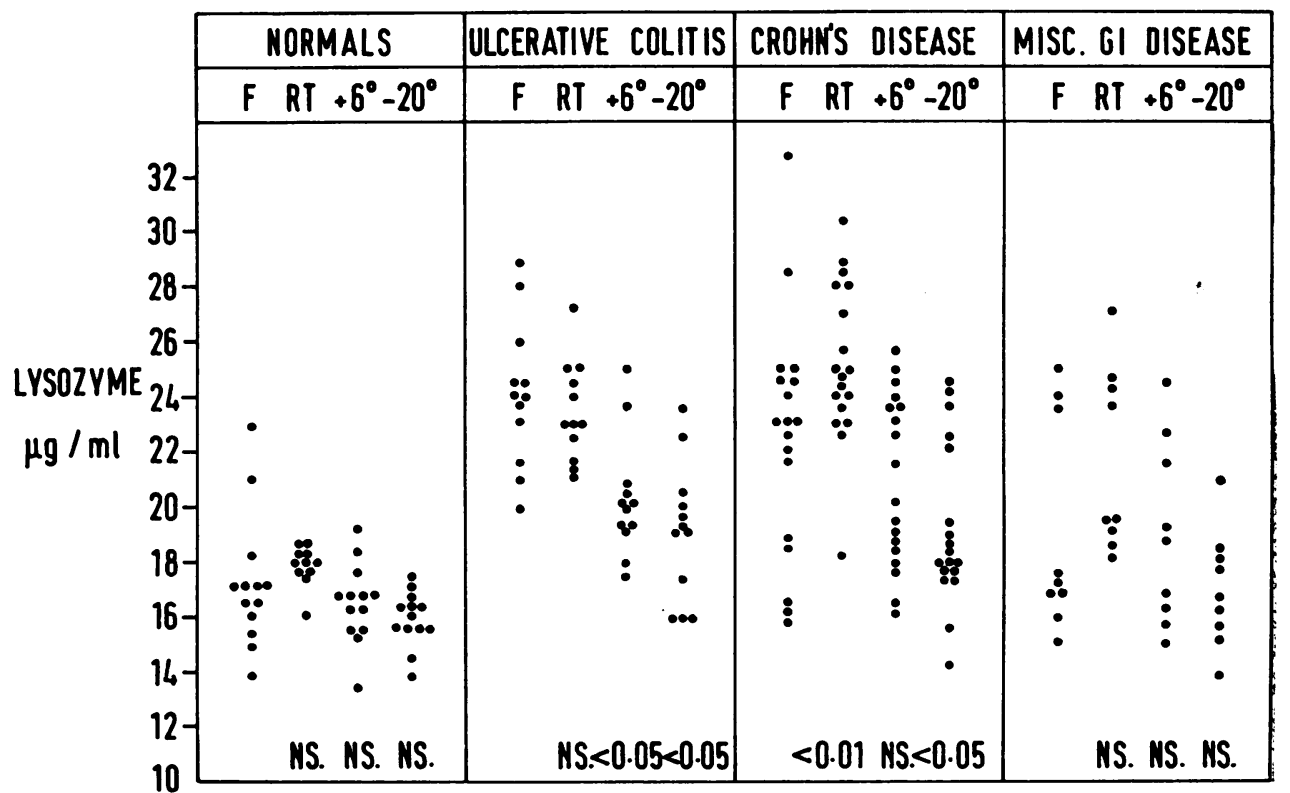

Fig. 1 Effects of four days' storage of serum lysozyme activity (lysoplate): $F$ = freshestimation; $R T /+6 /-20=$ values after storage at appropriate temperature for four days; $\mathrm{P}$ values refer to paired t tests in comparison with fresh value; $N S=$ not significant.

standards. When this 'calculated anionic activity' is plotted against 'total (lysoplate) activity' a direct linear relationship is found $(r=0.90, P<0.001$, $n=17$ ) (Fig. 4). In other words, the higher the lysoplate value the greater the anionic component.

The distance moved by the anionic zone was constant for each electrophoresis and appeared to have a mobility close to that of albumin.

(iii) Immunoelectrophoresis

No precipitate line could be demonstrated with serum, presumably a reflection of the low concentration of lysozyme in serum.

(iv) Radioimmunoelectrophoresis

Examination of autoradiographs showed clear evidence of lysozyme-antilysozyme precipitation in the expected site on the cathodal side of the origin but no such activity was found which would correspond to the site of anionic lysis.

\section{Discussion}

The present study has shown that estimates of SLA by lysoplates are influenced by the temperature at which serum is stored. In general, the lower the temperature of storage, the lower the value of SLA, the results being statistically significant in patients

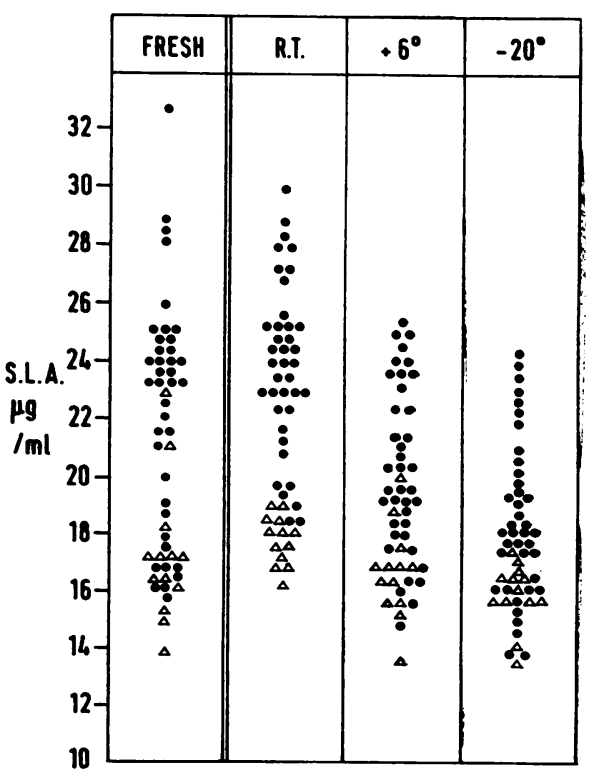

Fig. 2 Combined results from Fig. 1 to illustrate loss of separation of disease and control populations on storage at $-20^{\circ} \mathrm{C}$ for four days: $\triangle$ controls; $\bigcirc$ patients with inflammatory bowel disease and other gastrointestinal disease. 


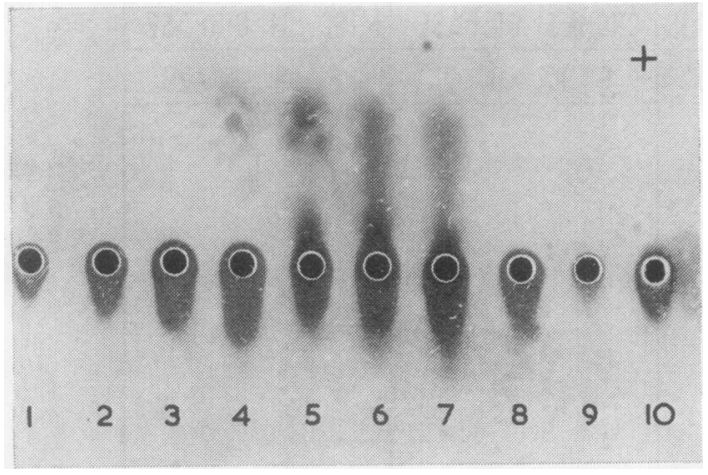

Fig. 3 Agarose-micrococcus plate after electrophoresis:

1 Egg white lysozyme, $10 \mathrm{\mu g} / \mathrm{ml}$

2 Egg white lysozyme, $50 \mu \mathrm{g} / \mathrm{ml}$

3 Egg white lysozyme, $100 \mathrm{\mu g} / \mathrm{ml}$

4 Purified human lysozyme

5 Serum sample-control

6 Serum sample-ulcerative colitis

7 Serum sample-Crohn's disease

8 Leucocyte extract

9 Rectal mucosal extract

10 Jejunal mucosal extract

+ positive electrode.

with inflammatory bowel disease. The overall effect of these changes due to freezing is to abolish the distinction between patient and control groups, which is apparent in the fresh and room temperature storage groups. The conditions of serum storage are, therefore, of considerable importance when comparing results of SLA estimations in different conditions and between different studies.

On electrophoresis serum contains an anionic lytic component in addition to the cationic lytic activity common to all lysozyme sources. This is apparent from the use of both an overlayer of micrococcus as an indicator after electrophoresis, and from electrophoresis through a suspension of micrococcus.

The cause of the anionic activity is not certain. The fact that it appeared to migrate with albumin, its linear relationship to 'total' SLA, and its slower development and weaker nature than the strong cationic lysis suggested the possibility of protein binding. This would be supported by the known avidity of egg white lysozyme for proteins (Alderton et al., 1945) and the formation of complexes of human lysozyme with lactoferrin (Perraudin et al., 1974), with IgG in myelomonocytic leukaemia (Finkle et al., 1973), and with $\alpha_{2}$-globulin in hypogammaglobulinaemia (Virella, 1975). A protein carrier has also been proposed to account for the differences in electrophoretic mobility of tear and salivary lysozyme (Revis, 1971). It is also known that

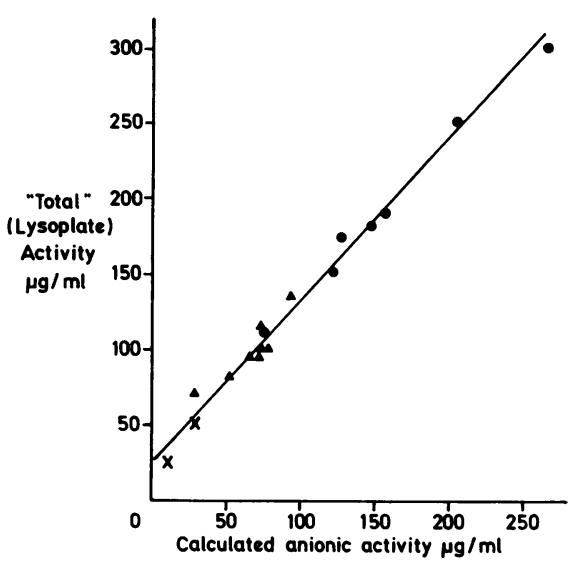

Fig. 4 Relationship of 'total' SLA as measured by lysoplate with value calculated for anionic component (determined from cationic value subtracted from lysoplate value) - calibrated from egg white standards: $\Delta$ controls; $\times$ controls diluted serum $1 / 4$ and $1 / 2$; - patients with inflammatory bowel disease.

egg white lysozyme added to (dog) serum produces a less than anticipated degree of lytic activity, and that human serum progressively diluted produces greater than anticipated lysis (Harrison and Swingler, 1971), these results being attributed to the influence of serum proteins.

However, the fact that no anionic activity was found on radioimmunoelectrophoresis does not support the concept that the anionic lysis is caused by lysozyme itself whether bound or not. Whatever its nature there is no doubt that it contributes to total serum lytic activity and must be considered as another variable in any assay dependent upon the lysis of Micrococcus lysodeikticus.

Since the original submission of this manuscript, Virella (1977) has described a similar technique of electrophoresis of lysozyme through a micrococcus suspension but makes no mention of any anionic lysis. There are methodological differences between our own and Virella's work, and further studies are required to decide whether these account for the different findings, and to determine the exact cause of the anionic lysis.

We have no proof that our two principal findings -the dual nature of SLA and its thermolability in inflammatory bowel disease-are related, although the increase in clarity of the anionic lytic zone with increased serum storage temperature suggests that this may be the case.

There are many factors which influence the lysis of $M$. lysodeikticus in agarose by serum lysozyme (Peeters and VanTrappen, 1977). The present study 
has identified two further sources of variability, the temperature of serum storage and the heterogeneous nature of serum lysozyme activity itself. Further studies are required to determine the exact cause of the anionic lysis and the clinical relevance of thermolability in inflammatory bowel disease.

We thank Drs W. Sircus and Anne Ferguson for permission to study patients under their care, and Dr G. Lidgard and Mrs Lynette Henderson for expert assistance. We are grateful to Messrs HoechstBehring for the kind gift of purified human lysozyme.

\section{References}

Alderton, G., Ward, W. H., and Fevold, H. L. (1945). Isolation of lysozyme from egg white. Journal of Biological Chemistry, 157, 43-58.

Dronfield, M. W., and Langman, M. J. S. (1975). Serum lysozyme in inflammatory bowel disease. Gut, 16, 985-987.

Falchuk, K. R., Perrotto, J. L., and Isselbacher, K. J. (1975). Serum lysozyme in Crohn's disease and ulcerative colitis. New England Journal of Medicine, 292, 395-397.

Finkle, H. I., Brownlow, K., and Elevitch, F. R. (1973). Monoclonal IgG-lysozyme (muramidase) complex in acute myelomonocytic leukaemia. American Journal of Clinical Pathology, 60, 936-940.

Harrison, J. F., and Swingler, M. (1971). The effect of serum macromolecules on the lysis of Micrococcus lysodeikticus cells by lysozyme. Clinica Chimica Acta, 31, 149-154.

Jolles, P. (1962). Lysozymes from rabbit spleen and dog spleen. In Methods in Enzymology, edited by S. P. Colowick and N. O. Kaplan, Vol. 5, p. 137. Academic Press, NewYork and London.

Lennard-Jones, J. E., Lockhart-Mummery, H. E., and
Morson, B. C. (1968). Clinical and pathological differentiation of Crohn's disease and proctocolitis. Gastroenterology, 54, 1162-1170.

Mallas, E., Terry, J. M., Asquith, P., and Cooke, W. T. (1976). Serum lysozyme in inflammatory bowel and coeliac disease. Journal of Clinical Pathology, 29, 598600.

Nugent, F. W., Mallari, R., George, H., and Ridley, N. (1976). Serum lysozyme in inflammatory bowel disease. Gastroenterology, 70, 1014-1016.

Osserman, E. F., Canfield, R. E., and Beychok, S. (1974). Lysozyme. Academic Press, New York and London.

Osserman, E. F., and Lawlor, D. P. (1966). Serum and urinary lysozyme (muramidase) in monocytic and monomyelocytic leukemia. Journal of Experimental Medicine, 124, 921-951.

Peeters, T. L., and VanTrappen, G. R. (1977). Factors influencing lysozyme determinations by the lysoplate method. Clinica Chimica Acta, 74, 217-225.

Peeters, T. L., VanTrappen, G., and Geboes, K. (1976). Serum lysozyme levels in Crohn's disease and ulcerative colitis. Gut, 17, 300-305.

Perraudin, J. P., Prieels, J. P., and Léonis, J. (1974). Interaction between lysozyme and some lactoferrin complex in human milk. Archives Internationales de Physiologie et de Biochimie, 82, 1001.

Revis, G. J. (1971). Immunoelectrophoretic identification of lysozyme in saliva. Proceedings of the Society for Experimental Biology and Medicine, 137, 90-96.

Schachter, H., and Kirsner, J. B. (1975). Definitions of inflammatory bowel disease of unknown etiology. Gastroenterology, 68, 591-600.

Virella, G. (1975). The electrophoretic mobility of serum lysozyme. Experientia, 31, 1465-1467.

Virella, G. (1977). Electrophoresis of lysozyme into micrococcus-containing agarose gel. Quantitative and analytical applications. Clinica Chimica Acta, 75, 107115. 